\title{
SEM, TEM, and AFM Analyses of Phase-Separated Polymer Blend Membranes for Gas Separations
}

\author{
I.H. Musselman ${ }^{1}$, N.P. Panapitiya ${ }^{1}$, D.K. Bushdiecker II ${ }^{1}$, M.P. Tomasek ${ }^{1}$, C.K. Miller ${ }^{1}$, C.J. Gilpin ${ }^{2}$, \\ K.J. Balkus, Jr. ${ }^{1}$, and J.P. Ferraris ${ }^{1}$ \\ ${ }^{1 .}$ Department of Chemistry, The University of Texas at Dallas, Richardson, TX 75080 \\ 2. Life Science Microscopy Facility, Purdue University, West Lafayette, IN 47907
}

Polymer blends are proving to be important in emerging technologies including as gas separation membranes [1]. The blending of existing polymers offers the distinct advantage of being able to develop new materials with potentially synergistic properties not attainable with the individual polymer components and without the need to synthesize new macromolecules [2]. We recently developed phaseseparated polymer blends for gas separation comprised of polybenzimidazole (PBI) and 6FDA-DAMDABA (6FDD) (Figure 1). In this system, PBI facilitates the separation of gas pairs and 6FDD enhances gas flux. Of critical importance is the blend interface, which determines the membrane's gas transport, mechanical, and adhesion properties [3]. Herein, we report the results of scanning electron microscopy (SEM), transmission electron microscopy (TEM), and atomic force microscopy (AFM) analyses of a PBI:6FDD (50:50) blend microstructure.

The blend membranes were fabricated by preparing separate solutions of commercial PBI and synthesized 6FDD [4] polymers in N,N-dimethylacetamide, combining them with stirring, and purging the excess solvent with nitrogen at $80{ }^{\circ} \mathrm{C}$. The concentrated solution was cast onto a glass substrate using a Sheen automatic applicator $(1133 \mathrm{~N})$ equipped with a doctor blade. The films were dried on a heated casting table under a nitrogen flow and then annealed under vacuum. Cross-section samples for SEM were prepared via freeze-fracture in liquid nitrogen, and thin sections for TEM and AFM were prepared using a Leica UC7 ultramicrotome with a Diatome Ultra diamond knife. The SEM samples were coated with $\mathrm{Au} / \mathrm{Pd}$ and imaged using a Zeiss SUPRA ${ }^{\circledR} 40$ SEM with a field emission gun operating at $10 \mathrm{keV}$. TEM images of the blend cross-section mounted on a c-flat holey carbon grid were acquired using a JEOL 1200 EX transmission electron microscope. AFM images were obtained using a Bruker Multimode 8 scanning probe microscope with NanoScope V controller in the PeakForce Tapping ${ }^{\mathrm{TM}}$ mode. A Young's modulus map and histogram for the polymer blend were acquired using PeakForce Quantitative Nanomechanical Mapping ${ }^{\mathrm{TM}}$ and the Derjaguin-Muller-Toporov model following required calibration $[5,6]$.

The SEM, TEM, and AFM images (Figure 2) show two distinct phases with a unique droplet - matrix morphology. The average droplet diameter was measured to be $1.46 \pm 0.60 \mu \mathrm{m}$. The identities of the dispersed droplet phase as 6FDD and the continuous matrix phase as PBI were confirmed by subjecting the membrane to Sohxlet extraction using tetrahydrofuran, which is known to selectively remove 6FDD. In the SEM image, a distinct gap is observed at the polymer blend interface, which is attributed to the freeze-fracture process. In contrast, no visible breaks or voids between the two phases were observed in TEM or AFM images of the sample cross-sections prepared using the ultramicrotome. The Young's modulus map for the polymer blend showed two components, and the histogram showed two distinct, but overlapping peaks (Figure 3). The local maximum for the PBI matrix (6 GPa) corresponds to the vendor supplied value of $5.9 \mathrm{GPa}$, and the peak at 3.2 GPa belongs to the 6FDD droplet phase [7]. 


\section{References:}

[1] LM Robeson, Polymer Blends: A Comprehensive Review (Carl Hanser Verlag, Munich) 2007.

[2] AI Isayev, Encylopedia of Polymer Blends. Vol. 1: Fundamentals (Wiley-VCH: Weinheim, Germany) 2010.

[3] M Stamm (ed.), Polymer Surfaces and Interfaces (Springer-Verlag: Berlin) 2008.

[4] IC Omole, SJ Miller and WJ Koros, Macromolecules 41 (2008), p. 6367.

[5] ME Dokukin and I Sokolov, Macromolecules 45 (2012), p. 4277.

[6] ME Dokukin and I Sokolov, Langmuir 28 (2012), p. 16060.

[7] The authors thank the National Science Foundation ([CBET] grants 0933563 and CHE-1126177 [for Bruker AVANCE IIITM 500 NMR]) and the U.S. Department of Energy (grants DE-NT0007636 and DE-FE0001293) for the financial support. The technical assistance of Dr. Helmut Gnaegi is gratefully acknowledged.<smiles>Cc1nc2cc(-c3ccc4nc(-c5cccc(C(C)(C)C)c5)[nH]c4c3)ccc2[nH]1</smiles><smiles>Cc1cc(C(=O)O)cc(N2C(=O)c3ccc(C(c4ccc5c(c4)C(=O)N(c4c(C)cc(C)c(C(C)(C)N6C(=O)c7ccc(C(C)(C)C(F)(F)F)cc7C6=O)c4C)C5=O)(C(F)(F)F)C(F)(F)F)cc3C2=O)c1</smiles>

Figure 1. Chemical structures of (left) PBI and (right) 6FDA-DAM:DABA.
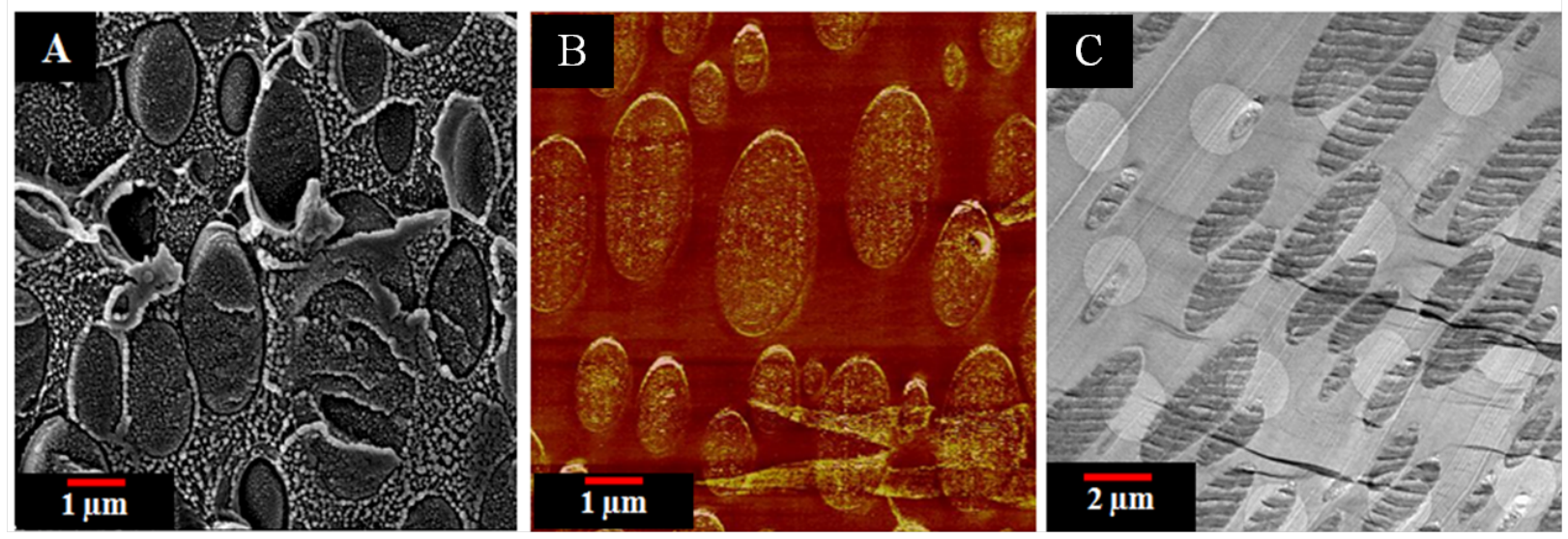

Figure 2. (A) SEM, (B) AFM, and (C) TEM images of PBI:6FDD (50:50) blend membrane crosssections.
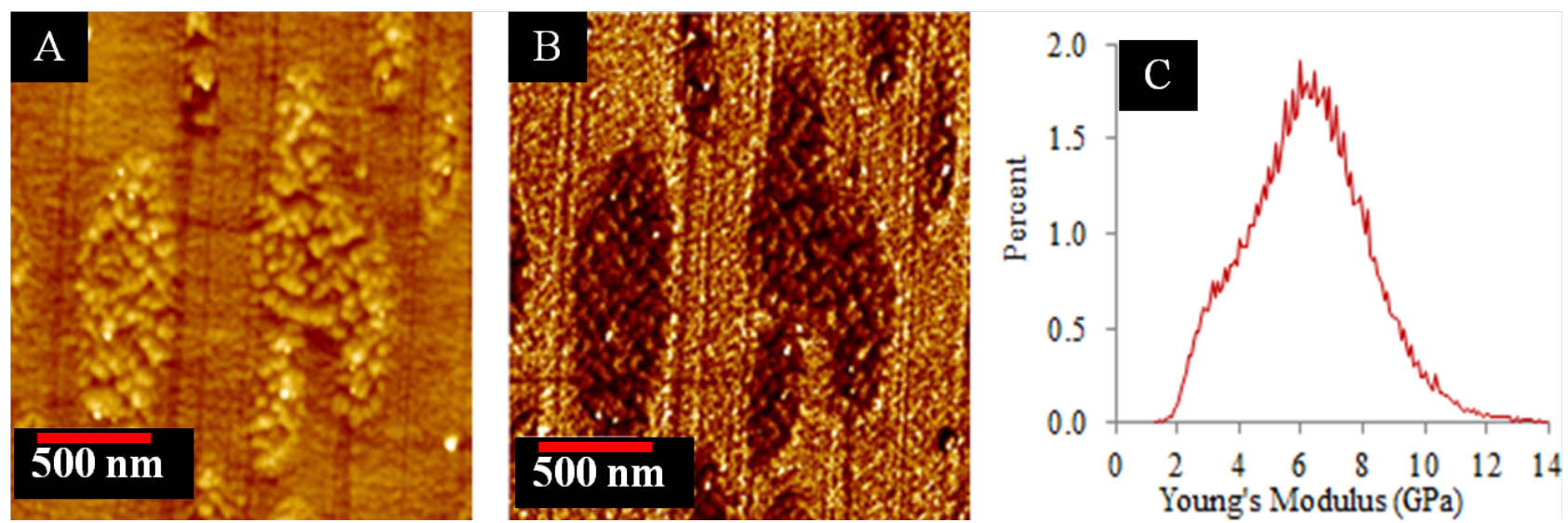

Figure 3. Atomic force microscopy (A) height image, (B) modulus map, and (C) modulus map histogram of a PBI:6FDD (50:50) blend membrane cross-section. 